PROCEEDINGS OF THE

AMERICAN MATHEMATICAL SOCIETY

Volume 136, Number 6, June 2008, Pages 2165-2176

S 0002-9939(08)09211-3

Article electronically published on February 14, 2008

\title{
ADJOINTS AND FORMAL ADJOINTS OF MATRICES OF UNBOUNDED OPERATORS
}

\author{
MANFRED MÖLLER AND FRANCISZEK HUGON SZAFRANIEC
}

(Communicated by Joseph A. Ball)

\begin{abstract}
In this paper we discuss diverse aspects of the mutual relationship between adjoints and formal adjoints of unbounded operators bearing a matrix structure. We emphasize the behaviour of row and column operators as they turn out to be the germs of an arbitrary matrix operator, providing most of the information about the latter as it is the troublemaker.
\end{abstract}

\section{INTRODUCTION}

In recent years, $2 \times 2$ matrices of unbounded operators have attracted considerable attention, roughly divided into two groups of problems: they occur as generators for semigroups, see 2], [13] and 4], and as tools in problems from Mathematical Physics, see [8], [5], 12]. The latter has attracted much interest in spectral properties of such matrices, in particular in its essential spectrum; see [1, 7], 10], 11] and the references therein.

Adjoints of operators bearing a matrix structure have been investigated case by case; it might be difficult to find any general approach to the problem when there are rows or columns with more than one unbounded entry. So as to mention some partial results, let us refer to [6] and [14] where the only nonzero entries are those off the diagonal. On the other hand, in [3] and [13] examples are given showing that the adjoint of a matrix operator $A$ and its formal adjoint $A^{\times}$, that is, the matrix of adjoints of all the particular entries, may be quite different. This supports the idea of the present paper to build a common framework for all the cases. Positive results in this matter are intertwined with counterexamples; the latter indicate that $A^{\times}$ may not contain enough information on $A^{\prime}$ itself. Thus, what is essentially trivial for bounded operators appears to become erratic for unbounded operators.

It turns out that the study of row and column operators separately is pretty much helpful as a matrix can be decomposed in a sense by means of these two. Indeed, column and row operators have a more predictable behaviour, which helps to understand the 2 by 2 matrix case; this case is interesting as well as difficult enough to deserve a careful treatment. The pith of the problem can be simply described by saying that

Received by the editors December 11, 2006, and, in revised form, April 27, 2007.

2000 Mathematics Subject Classification. Primary 47A05; Secondary 47D06.

Key words and phrases. Operator matrix, row operator, column operator, adjoint and formal adjoint.

This work was supported by a grant of the NRF of South Africa, GUN 2053746, and by the grant KBN 2 P 03A 637024 (Poland). 
if $A$ is either a row or a column operator (even in a Hilbert space) with both entries being unbounded and closed, then it is rather unlikely that $A$ will coincide with its second adjoint.

On the other hand, it is important to stress that if a column has only one entry which is unbounded, then the problem does not appear at all; this is the most frequent case which occurs in the literature.

\section{Preliminaries}

Reasonable assumptions on $2 \times 2$ operator matrices are that their entries are closed densely defined operators and that the operators they determine are densely defined. However, when one divides bigger operator matrices into $2 \times 2$ blocks, the blocks in the latter structure will not be closed, in general. Matrices of arbitrary size occur in an attempt to determine normal extensions of unbounded operators, see [15], and our intention is to elaborate somewhere else on these kinds of matrices from the point of view of the present paper. Indeed, also for genuine $2 \times 2$ operator matrices, the blocks are often not assumed to be closed as the closedness of at least some of the blocks would be pointless anyway.

Henceforth, for $i=1,2$, let $E_{i}$ and $F_{i}$ be locally convex Hausdorff spaces and for $i, j=1,2$, let $A_{i j} \in \mathfrak{L}\left(E_{j}, F_{i}\right)$, where $\mathfrak{L}\left(E_{j}, F_{i}\right)$ denotes the set of all densely defined operators from $E_{j}$ to $F_{i}$. The closed densely defined and continuous everywhere defined operators in these spaces are denoted by $\mathfrak{C}\left(E_{j}, F_{i}\right)$ and $\mathfrak{B}\left(E_{j}, F_{i}\right)$, respectively. Finite direct sums will be denoted with the symbol $\oplus$, meaning that in the case of Hilbert spaces, the direct sum will always be identified with the orthogonal one. Define

$$
A=\left(\begin{array}{ll}
A_{11} & A_{12} \\
A_{21} & A_{22}
\end{array}\right)
$$

from $E$ to $F$, where $E=E_{1} \oplus E_{2}$ and $F=F_{1} \oplus F_{2}$, and the domain of $A$ is given by

$$
\mathcal{D}(A)=\left(\mathcal{D}\left(A_{11}\right) \cap \mathcal{D}\left(A_{21}\right)\right) \oplus\left(\mathcal{D}\left(A_{12}\right) \cap \mathcal{D}\left(A_{22}\right)\right) .
$$

The range of $A$ will be denoted by $\mathcal{R}(A)$.

Here we will only consider the case that $A$ is densely defined, so we require that

$$
\mathcal{D}\left(A_{1 j}\right) \cap \mathcal{D}\left(A_{2 j}\right) \text { is dense in } E_{j} \text { for } j=1,2 .
$$

In particular, since all $A_{i j}$ are densely defined, their adjoints $A_{i j}^{\prime}$ must necessarily be closed operators from $F_{i}^{\prime}$ to $E_{j}^{\prime}$, and we can define the operator

$$
A^{\times}=\left(\begin{array}{ll}
A_{11}^{\prime} & A_{21}^{\prime} \\
A_{12}^{\prime} & A_{22}^{\prime}
\end{array}\right)
$$

from $F^{\prime}$ to $E^{\prime}$, where, according to (2.1),

$$
\mathcal{D}\left(A^{\times}\right)=\left(\mathcal{D}\left(A_{11}^{\prime}\right) \cap \mathcal{D}\left(A_{12}^{\prime}\right)\right) \oplus\left(\mathcal{D}\left(A_{21}^{\prime}\right) \cap \mathcal{D}\left(A_{22}^{\prime}\right)\right) .
$$

Row and column operators of arbitrary size are defined as follows. A row operator $R \stackrel{\text { def }}{=} R_{R_{1}, \ldots, R_{n}}$ is a linear mapping defined in $\bigoplus_{j=1}^{n} E_{j}$, taking values in $E_{0}$ and acting according to

$$
R\left(\bigoplus_{j=1}^{n} f_{j}\right)=\sum_{j=1}^{n} R_{j} f_{j}, \quad \bigoplus_{j=1}^{n} f_{j} \in \mathcal{D}(R) \stackrel{\text { def }}{=} \bigoplus_{j=1}^{n} \mathcal{D}\left(R_{j}\right),
$$


where $E_{0}, E_{1}, \ldots, E_{n}$ are locally convex Hausdorff spaces, and the $R_{j}$ are linear operators from $E_{j}$ to $E_{0}$. Clearly, $R$ is densely defined if and only if all $R_{j}$ are densely defined.

A column operator $C \stackrel{\text { def }}{=} C_{C_{1}, \ldots, C_{n}}$ is a linear mapping defined in $F_{0}$, taking values in $\bigoplus_{j=1}^{n} F_{j}$ and acting as

$$
\mathcal{D}(C) \stackrel{\text { def }}{=} \bigcap_{j=1}^{n} D\left(C_{j}\right), \quad C f \stackrel{\text { def }}{=} \bigoplus_{j=1}^{n} C_{j} f, \quad f \in \mathcal{D}(C),
$$

the spaces $F_{0}, F_{1}, \ldots, F_{n}$ are locally convex Hausdorff spaces, and $C_{j} \in \mathfrak{L}\left(F_{0}, F_{j}\right)$ for $j=1, \ldots, n$. To such a column operator we associate the row operator

$$
C^{\times} \stackrel{\text { def }}{=} R_{C_{1}^{\prime}, \ldots, C_{n}^{\prime}}
$$

which may not be densely defined even if $C$ is densely defined.

The important notice we can make at this stage more precise is that row operators behave differently from column operators. In particular, supposing all the entries of $R$ are closed,

$$
R^{\prime \prime} \text { may not be equal to } R \text { though always } R^{\prime \times}=R \text {. }
$$

Needless to say a similar behaviour concerns column operators. This outlines once more the flavour of our paper.

In Hilbert spaces, we will take the usual Hilbert space adjoints of operators, i. e., with respect to the sesquilinear scalar product rather than the adjoints with respect to bilinear forms for dual pairs. Since the results of this paper are independent of whether the duality is realized by bilinear forms or sesquilinear forms, in Hilbert space we will always use Hilbert space adjoints. With some abuse of notation, we shall write e. g. $C^{\times}$in both cases, where its meaning will be clear from the context. This is applicable in particular to Krĕn space adjoints if each of the component spaces is a Kreln space, because in this case the fundamental symmetry on the direct sum is the direct sum of the fundamental symmetries on the components.

\section{ROW OPERATORS}

In this section let $R \stackrel{\text { def }}{=} R_{R_{1}, \ldots, R_{n}}$ be a row operator as defined above.

Proposition 3.11 The adjoint $R^{\prime}$ of a densely defined row operator $R$ is the column operator formed by the adjoints of the $R_{j}$, that is,

$$
R^{\prime}=C_{R_{1}^{\prime}, \ldots, R_{n}^{\prime}}
$$

Proof. To show $R^{\prime} \subset C_{R_{1}^{\prime}, \ldots, R_{n}^{\prime}}$ let all $f_{j} \in \mathcal{D}\left(R_{j}\right)$ and $g \in \mathcal{D}\left(R^{\prime}\right)$. Then

$$
\left\langle\bigoplus_{j} f_{j}, R^{\prime} g\right\rangle=\left\langle\sum_{j} R_{j} f_{j}, g\right\rangle=\sum_{j}\left\langle R_{j} f_{j}, g\right\rangle
$$

Putting $f_{j}=0$ for $j \neq k$, it follows that $g \in \mathcal{D}\left(R_{k}^{\prime}\right)$ for each $k=1, \ldots, n$ and $\left\langle R_{k} f_{k}, g\right\rangle=\left\langle f_{k}, R_{k}^{\prime} g\right\rangle$. Thus

$$
\left\langle\bigoplus_{j} f_{j}, R^{\prime} g\right\rangle=\left\langle\bigoplus_{j} f_{j}, \bigoplus_{j} R_{j}^{\prime} g\right\rangle,
$$

which proves the inclusion " $\subset$ ".

\footnotetext{
${ }^{1}$ Propositions 104.1 and 4.5 have been proved for linear relations in Hilbert spaces in 9 , Proposition 2.1.
} 
Conversely, let $g \in \mathcal{D}\left(C_{R_{1}^{\prime}, \ldots, R_{n}^{\prime}}\right)=\bigcap_{j=1}^{n} \mathcal{D}\left(R_{j}^{\prime}\right)$. Then, for all $f_{j} \in \mathcal{D}\left(R_{j}\right)$, $\left\langle f_{j}, R_{j}^{\prime} g\right\rangle=\left\langle R_{j} f_{j}, g\right\rangle$, and thus

$$
\left\langle\bigoplus_{j} f_{j}, \bigoplus_{j} R_{j}^{\prime} g\right\rangle=\left\langle\sum_{j} R_{j} f_{j}, g\right\rangle=\left\langle R\left(\bigoplus_{j} f_{j}\right), g\right\rangle,
$$

which shows $g \in \mathcal{D}\left(R^{\prime}\right)$.

Remark 3.2. The simplest example showing that a row operator with closed entries may not be even closable is to consider $R_{1}$ and $R_{2}$ selfadjoint with $\mathcal{D}\left(R_{1}\right) \cap \mathcal{D}\left(R_{2}\right)=$ $\varnothing$. Then, according to Proposition \, $\mathcal{D}\left(R^{\prime}\right)=\mathcal{D}\left(R_{1}^{\prime}\right) \cap \mathcal{D}\left(R_{2}^{\prime}\right)=\varnothing$; hence $R$ is not closable. This is a rather extreme example in a sense; a richer one concerning the same question will be given below.

Example 3.3. Define $R_{1}$ and $R_{2}$ in $\ell^{2}$ as follows:

$$
R_{1} e_{k} \stackrel{\text { def }}{=} k^{2} e_{k}, \quad R_{2} e_{k} \stackrel{\text { def }}{=} k e_{0}+k^{2} e_{k}, \quad k=0,1,2, \ldots,
$$

where $\left(e_{n}\right)_{n=0}^{\infty}$ is the orthodox zero-one orthonormal basis of $\ell^{2}$. To properly establish $R_{1}$ and $R_{2}$, we define their domains by

$$
\begin{aligned}
& \mathcal{D}\left(R_{1}\right)=\left\{f=\sum_{k=0}^{\infty} \gamma_{k} e_{k}: \sum_{k=0}^{\infty}\left|\gamma_{k} k^{2}\right|^{2}<\infty\right\}, \\
& \mathcal{D}\left(R_{2}\right)=\left\{f=\sum_{k=0}^{\infty} \gamma_{k} e_{k} \in \mathcal{D}\left(R_{1}\right): \sum_{k=0}^{\infty} \gamma_{k} k \text { converges }\right\} .
\end{aligned}
$$

Then, as usual, the operators $R_{i}$ are defined as

$$
R_{i} f=\sum_{k=0}^{\infty} \gamma_{k} R_{i} e_{k}, \quad f=\sum_{k=0}^{\infty} \gamma_{k} e_{k} \in \mathcal{D}\left(R_{i}\right) .
$$

Because $R_{1}$ is a diagonal operator on its maximal domain, it is closed. In order to prove the closedness of $R_{2}$ let

$$
f_{n}=\sum_{k=0}^{\infty} \gamma_{n k} e_{k} \in \mathcal{D}\left(R_{2}\right), \quad f_{n} \rightarrow f=\sum_{k=0}^{\infty} \gamma_{k} e_{k}, \text { and } \quad R_{2} f_{n} \rightarrow g=\sum_{k=0}^{\infty} \delta_{k} e_{k} .
$$

If $P$ denotes the orthogonal projection of the Hilbert space onto the closed linear span of $\left\{e_{k}\right\}_{k=1}^{\infty}$, then $P R_{2} f_{n}=R_{1} f_{n}$, and so $R_{1} f_{n} \rightarrow P g$. Since $R_{1}$ is closed, it follows that $f \in \mathcal{D}\left(R_{1}\right)$ and $P g=R_{1} f$.

Note that

$$
\begin{gathered}
\sum_{k=1}^{\infty}\left|\gamma_{n k} k^{2}-\gamma_{k} k^{2}\right|^{2}=\left\|R_{1} f_{n}-R_{1} f\right\|^{2} \rightarrow 0 \quad \text { as } \quad n \rightarrow \infty, \\
(I-P) R_{2} f_{n}=\sum_{k=1}^{\infty} \gamma_{n k} k e_{0}
\end{gathered}
$$

and that $R_{2} f_{n} \rightarrow g$ implies

$$
\sum_{k=1}^{\infty} \gamma_{n k} k \rightarrow \delta_{1} \quad \text { as } \quad n \rightarrow \infty
$$


Since for $m \geq 1$ and $n=0,1, \ldots$,

$$
\begin{gathered}
\left|\sum_{k=0}^{m} \gamma_{k} k-\delta_{1}\right| \leq\left|\sum_{k=0}^{m} \gamma_{n k} k-\delta_{1}\right|+\sum_{k=0}^{m}\left|\gamma_{n k} k-\gamma_{k} k\right| \\
\leq\left|\sum_{k=0}^{m} \gamma_{n k} k-\delta_{1}\right|+\left(\sum_{k=1}^{\infty}\left|\gamma_{n k} k^{2}-\gamma_{k} k^{2}\right|^{2}\right)^{\frac{1}{2}}\left(\sum_{k=1}^{\infty}|k|^{-2}\right)^{\frac{1}{2}},
\end{gathered}
$$

from (3.1) and (3.2), we get that for each $\varepsilon>0$ there is $n \in \mathbb{N}$ such that

$$
\left|\sum_{k=0}^{m} \gamma_{k} k-\delta_{1}\right| \leq\left|\sum_{k=m+1}^{\infty} \gamma_{n k} k\right|+\varepsilon
$$

for $m \geq 1$. From (3.3) and the fact that $\sum_{k=1}^{\infty} \gamma_{n k} k$ converges, we deduce that

$$
\left|\sum_{k=1}^{m} \gamma_{k} k-\delta_{1}\right| \leq 2 \varepsilon
$$

for sufficiently large $m$, which proves that $\sum_{k=1}^{\infty} \gamma_{k} k$ converges to $\delta_{1}$. This shows $f \in \mathcal{D}\left(R_{2}\right)$ and $g=R_{2} f$.

Finally, letting for $n \geq 1, f_{n}=n^{-1} e_{n}$, we have $f_{n} \in \mathcal{D}\left(R_{2}\right), f_{n} \rightarrow 0$ as $n \rightarrow \infty$, and

$$
R_{1}\left(-f_{n}\right)+R_{2} f_{n}=n n^{-1} e_{0}=e_{0} .
$$

So $R$ is not closable. However the linear span of $\left(e_{n}\right)_{n=0}^{\infty}$ is included in $\mathcal{D}\left(R_{1}\right) \cap$ $\mathcal{D}\left(R_{2}\right)$ as well as in $\mathcal{D}\left(R_{1}^{\prime}\right) \cap \mathcal{D}\left(R_{2}^{\prime}\right)$, and this is what makes this example more interesting than that argued for in Remark 3.2 .

Corollary 3.4. For $R$ to be closable it is necessary but not sufficient that $R_{1}, \ldots$, $R_{n}$ are closable.

Proof. Use all of the above and the fact, which is implicit in Proposition 1, that $\mathcal{D}\left(R^{\prime}\right)=\bigcap_{j=1}^{n} \mathcal{D}\left(R_{j}^{\prime}\right)$.

Note that in general $R$ is not closed even if all its entries are closed. However, we trivially have

Proposition 3.5. Assume that at most one of the entries of $R$ is not bounded. Then $R$ is closed (closable) if and only if all $R_{j}$ are closed (closable). In this case $R^{\prime}$ is densely defined.

In the following we have a particular result when the closure of $R$ can be determined explicitly.

Proposition 3.6. Let $n=2$, assume that $R_{1}$ is injective, that $\mathcal{R}\left(R_{2}\right) \subset \mathcal{R}\left(R_{1}\right)$ and that $R_{1}^{-1} R_{2}$ is an operator with a bounded extension $K \in \mathfrak{B}\left(E_{2}, E_{1}\right)$. Then

$$
\bar{R}=\left(\overline{R_{1}}, 0\right)\left(\begin{array}{cc}
I & K \\
0 & I
\end{array}\right),
$$

and $\mathcal{D}(\bar{R})=\left\{f \oplus g \in E_{1} \oplus E_{2}: f+K g \in \mathcal{D}\left(\overline{R_{1}}\right)\right\}$.

Proof. The operator $\hat{R}=\left(\overline{R_{1}}, 0\right)\left(\begin{array}{cc}I & K \\ 0 & I\end{array}\right)$ is closed since the $2 \times 2$ matrix operator on the right is invertible. Also, for $f \in \mathcal{D}\left(R_{1}\right), g \in \mathcal{D}\left(R_{2}\right)$ we have $K g=R_{1}^{-1} R_{2} g \in$ $\mathcal{D}\left(R_{1}\right)$ and thus $f+K g \in \mathcal{D}\left(R_{1}\right)$, and

$$
\hat{R}(f \oplus g)=\left(\overline{R_{1}}, 0\right)\left(f+R^{-1} R_{2} g\right) \oplus g=R_{1} f+R_{2} g=R(f \oplus g) .
$$


This shows that $R \subset \hat{R}$ and thus $\bar{R} \subset \hat{R}$ as $\hat{R}$ is closed. We calculate

$$
\hat{R}^{\prime}=\left(\begin{array}{cc}
I & 0 \\
K^{\prime} & I
\end{array}\right)\left(\begin{array}{c}
R_{1}^{\prime} \\
0
\end{array}\right)=\left(\begin{array}{c}
R_{1}^{\prime} \\
K^{\prime} R_{1}^{\prime}
\end{array}\right) .
$$

From $R_{2} \subset R_{1} K$ it follows that $K^{\prime} R_{1}^{\prime} \subset\left(R_{1} K\right)^{\prime} \subset R_{2}^{\prime}$. In particular, $\mathcal{D}\left(R_{1}^{\prime}\right) \subset$ $\mathcal{D}\left(R_{2}^{\prime}\right)$ and hence $K^{\prime} R_{1}^{\prime}=R_{2}^{\prime}$ on $\mathcal{D}\left(R_{1}^{\prime}\right)$. Thus $\hat{R}^{\prime}=R^{\prime}$ by (3.4) and Proposition 1 which proves $\hat{R}=\bar{R}$ since $\hat{R}$ is closed. The representation of $\mathcal{D}(\bar{R})$ is now obvious from the representation of $\bar{R}$.

\section{Column operators}

In this section, let $C \stackrel{\text { def }}{=} C_{C_{1}, \ldots, C_{n}}$ be a densely defined column operator.

Proposition 4.1. $C^{\times} \subset C^{\prime}$.

Proof. Since $C$ is densely defined, $C^{\prime}$ is an operator. Let $g_{j} \in \mathcal{D}\left(C_{j}^{\prime}\right), f \in \mathcal{D}(C)$. Then

$$
\left\langle f, C^{\times}\left(\bigoplus_{j} g_{j}\right)\right\rangle=\left\langle f, \sum_{j} C_{j}^{\prime} g_{j}\right\rangle=\sum_{j}\left\langle f, C_{j}^{\prime} g_{j}\right\rangle=\sum_{j}\left\langle C_{j} f, g_{j}\right\rangle=\left\langle C f, \bigoplus_{j} g_{j}\right\rangle .
$$

This proves $\bigoplus_{j} g_{j} \in \mathcal{D}\left(C^{\prime}\right)$ and $C^{\prime}\left(\bigoplus_{j} g_{j}\right)=C^{\times}\left(\bigoplus_{j} g_{j}\right)$.

Referring back to the preceding section, let us recall that $R^{\times}=C_{R_{1}^{\prime}, \ldots, R_{n}^{\prime}}$. Then the conclusion of Proposition 1 can be restated as

$$
R^{\prime}=R^{\times} \text {. }
$$

Now double applications of this and Proposition 4.1 lead to

Corollary 4.2. For a row operator $R$ and a column operator $C$ we have

$$
R^{\prime \prime}=R^{\times \prime} \supset R^{\times \times}=R^{\prime \times}, \quad C^{\prime \prime} \subset C^{\times \prime}=C^{\times \times} .
$$

Proposition 4.3. $1^{\circ} C$ is closed if $C_{1}, \ldots, C_{n}$ are closed. $2^{\circ} C$ is closable if $C_{1}, \ldots, C_{n}$ are closable. $3^{\circ} \overline{C^{\times}}=C^{\prime}$ if and only if $\bar{C}=C_{\overline{C_{1}}, \ldots, \overline{C_{n}}}$.

Proof. $1^{\circ}$ is straightforward, and $2^{\circ}$ is an immediate consequence of $1^{\circ}$. From Corollary 4.2 we get

$$
\overline{C^{\times}}=C^{\prime} \Longleftrightarrow C^{\times \prime}=C^{\prime \prime} \Longleftrightarrow C^{\times \times}=C^{\prime \prime} .
$$

Then the conclusion follows by observing that $C^{\prime \prime}=\bar{C}$ and

$$
C^{\times \times}=R_{C_{1}^{\prime}, \ldots, C_{n}^{\prime}}^{\times}=C_{\overline{C_{1}}, \ldots, \overline{C_{n}}} .
$$

Remark 4.4. The sufficient condition for $C$ to be closable, which is in Proposition 4.3. $2^{\circ}$, turns out to be not necessary. For this let $H_{1}$ and $H_{2}$ be Hilbert spaces and $C \in \mathfrak{C}\left(H_{1}, H_{2}\right) \backslash \mathfrak{B}\left(H_{1}, H_{2}\right)$, that is, $\mathcal{D}\left(C^{*}\right) \neq H_{2}$. Let $x \in H_{2} \backslash \mathcal{D}\left(C^{*}\right), P$ be the orthogonal projection onto the span of $x, C_{1}=P C, C_{2}=(I-P) C$. Then $C=\left(\begin{array}{l}C_{1} \\ C_{2}\end{array}\right)$ with $C_{1}$ not closable. Here, with some abuse of notation, the range spaces of $C_{1}$ and $C_{2}$ are $\mathcal{R}(P)$ and $\mathcal{R}(I-P)$, respectively.

Indeed, assume that $x \in \mathcal{D}\left(C_{1}^{*}\right)$. Then we have for $f \in \mathcal{D}(C)$ that

$$
\langle C f, x\rangle=\langle C f, P x\rangle=\langle P C f, x\rangle=\left\langle f, C_{1}^{*} x\right\rangle,
$$

and the contradiction $x \in \mathcal{D}\left(C^{*}\right)$ would follow. Since $\mathcal{D}\left(C_{1}^{*}\right) \subset \mathcal{R}(P)$ and $\mathcal{R}(P)$ is one-dimensional, $\mathcal{D}\left(C_{1}^{*}\right)=\{0\}$ follows. Thus $C_{1}$ is not closable. 
For other examples with nonclosable $C_{1}$ but closable $C_{2}$ and $C$ we refer to 3 , Sections 2 and 3] and [13, Section 1].

Proposition 4.5. Assume that at most one of the entries of $C$ does not satisfy $C_{j} \in \mathfrak{B}\left(F_{0}, F_{j}\right)$. Then $C^{\times}=C^{\prime}$.

Proof. Obviously, $\bar{C}=C_{\overline{C_{1}}, \ldots, \overline{C_{2}}}$, and hence $\overline{C^{\times}}=C^{\prime}$ by Proposition $4.33^{\circ}$. Clearly, $C^{\times}$satisfies the assumptions of Proposition 3.5. and thus $C^{\times}$is closed.

In the following result, $C^{\times}$and $C^{\prime}$ are given explicitly; in particular, the structure of their domains is transparent.

Proposition 4.6. Let $C_{1}, C_{2}$ be such that $\mathcal{D}\left(C_{1}\right)=\mathcal{D}\left(C_{2}\right)$ and assume that $C_{1}$ is injective with $C_{2} C_{1}^{-1}$ having an extension $K \in \mathfrak{B}\left(F_{1}, F_{2}\right)$. Then

$$
C^{\times}=\left(C_{1}^{\prime}, C_{1}^{\prime} K^{\prime}\right) \quad \text { and } \quad C^{\prime}=\left(C_{1}^{\prime}, 0\right)\left(\begin{array}{cc}
I & K^{\prime} \\
0 & I
\end{array}\right) .
$$

In particular, $\mathcal{D}\left(C^{\times}\right)=\left\{f \oplus g \in F_{1}^{\prime} \oplus F_{2}^{\prime}: f \in \mathcal{D}\left(C_{1}^{\prime}\right), K^{\prime} g \in \mathcal{D}\left(C_{1}^{\prime}\right)\right\}$ and $\mathcal{D}\left(C^{\prime}\right)=$ $\left\{f \oplus g \in F_{1}^{\prime} \oplus F_{2}^{\prime}: f+K^{\prime} g \in \mathcal{D}\left(C_{1}^{\prime}\right)\right\}$.

Proof. Since $C_{2}=C_{2} C_{1}^{-1} C_{1}=K C_{1}, C_{2}^{\prime}=C_{1}^{\prime} K^{\prime}$, and the statements about $C^{\times}$ follow.

For $C$ we have the representation

$$
C=\left(\begin{array}{c}
C_{1} \\
K C_{1}
\end{array}\right)=\left(\begin{array}{cc}
I & 0 \\
K & I
\end{array}\right)\left(\begin{array}{c}
C_{1} \\
0
\end{array}\right)
$$

which gives

$$
C^{\prime}=\left(C_{1}^{\prime}, 0\right)\left(\begin{array}{cc}
I & K^{\prime} \\
0 & I
\end{array}\right) \text {. }
$$

The statement about the domain of $C^{\prime}$ immediately follows from this.

\section{More about column operators in Hilbert spaces}

Here we assume that $F_{0}, F_{1}, F_{2}$ are Hilbert spaces and that $C=C_{C_{1}, C_{2}}$ is a densely defined closable column operator. Let $D_{0}$ be a subspace of $F_{0}$ satisfying

$$
D_{0} \subset \mathcal{D}\left(\bar{C} C^{\times}\right) .
$$

Notice that, if $\overline{C^{\times}}=C^{*}$, then, by part $3^{\circ}$ of Proposition 4.3,

$$
\bar{C} C^{\times}=\left(\begin{array}{ll}
\overline{C_{1}} C_{1}^{*} & \overline{C_{1}} C_{2}^{*} \\
\overline{C_{2}} C_{1}^{*} & \overline{C_{2}} C_{2}^{*}
\end{array}\right) .
$$

We want to investigate the following questions 2

- when is $D_{0}$ a core for $C^{*}$ ?

- when is $D_{0}$ a core for $C^{\times}$?

Proposition 5.1. Let $D_{1}$ be a subspace of $F_{0}$ such that $D_{0} \subset D_{1} \subset \mathcal{D}\left(C^{*}\right)$. Then $D_{0}$ is a core for $\left.C^{*}\right|_{D_{1}}$ if and only if

$$
\left(\left(I+\bar{C} C^{\times}\right)\left(D_{0}\right)\right)^{\perp} \cap D_{1}=\{0\} .
$$

\footnotetext{
${ }^{2} \mathcal{D} \subset \mathcal{D}(A)$ is a core for a closable operator $A$ if $A \subset \overline{\left.A\right|_{\mathcal{D}}}$.
} 
Proof. Due to (5.1), for $f \in D_{1}$ to belong to the left-hand side of (5.2) means precisely that

$$
0=\left\langle f,\left(I+\bar{C} C^{*}\right) g\right\rangle \text { for all } g \in D_{0} .
$$

Because $D_{1} \subset \mathcal{D}\left(C^{*}\right)$, this is what is required for $D_{1}$ to be a core for $C^{*}$.

Proposition 5.2. Let $C_{1}, C_{2}$ be such that $\mathcal{D}\left(C_{1}\right)=\mathcal{D}\left(C_{2}\right)$ and assume that $C_{1}$ is injective with $C_{2} C_{1}^{-1}$ having an extension $K \in \mathfrak{B}\left(F_{1}, F_{2}\right)$. Let $D_{0}$ be a subspace of $\mathcal{D}\left(C^{*}\right)$. Also assume that there is at least one $v_{0} \in F_{2} \backslash\{0\}$ such that $\left(-K^{*} v_{0}, v_{0}\right) \in$ $D_{0}$. Then $D_{0}$ is a core for $C^{*}$ if and only if $D_{0}$ is dense in $F_{1} \oplus F_{2}$ and $\left(I, K^{*}\right)\left(D_{0}\right) \cap$ $\mathcal{D}\left(C_{1}^{*}\right)$ is a core for $C_{1}^{*}$.

Proof. Since we assume that $C$ is closable, $C^{*}$ is densely defined, and the condition that $D_{0}$ is dense in $F_{1} \oplus F_{2}$ is necessary for a core. Thus we may assume this property for the remainder of the proof. Using the graph norm of $C^{*}$ as in the proof of Proposition 5.1, we have to investigate when, for $f \oplus g \in \mathcal{D}\left(C^{*}\right)$,

$$
\langle u \oplus v, f \oplus g\rangle+\left\langle C^{*}(u \oplus v), C^{*}(f \oplus g)\right\rangle=0
$$

for all $u \oplus v \in D_{0}$ implies $f \oplus g=0$. By Proposition 4.6.

$$
C^{*}(u \oplus v)=C_{1}^{*}\left(u+K^{*} v\right) \text { and } C^{*}(f \oplus g)=C_{1}^{*}\left(f+K^{*} g\right) .
$$

Introducing $w=u+K^{*} v$ and $h=f+K^{*} g$, (5.3) can be written as

$$
\langle u, f\rangle+\langle v, g\rangle+\left\langle C_{1}^{*} w, C_{1}^{*} h\right\rangle=0,
$$

which is the same as

$$
\left\langle w-K^{*} v, h-K^{*} g\right\rangle+\langle v, g\rangle+\left\langle C_{1}^{*} w, C_{1}^{*} h\right\rangle=0 .
$$

Note that

$$
\left\langle w-K^{*} v, h-K^{*} g\right\rangle+\langle v, g\rangle-\langle w, h\rangle=\left\langle\left(I+K K^{*}\right) v-K w, g\right\rangle-\left\langle K^{*} v, h\right\rangle .
$$

First we want to find a condition for $h=0$; thus we may modify $f$ and $g$ as long as $f+K^{*} g=h$. If $\left(I+K K^{*}\right) v \neq K w$ choose $g \in F_{2}$ such that

$$
\left\langle\left(I+K K^{*}\right) v-K w, g\right\rangle=\left\langle K^{*} v, h\right\rangle .
$$

If $\left(I+K K^{*}\right) v=K w$, we replace $u \oplus v$ with $\left(u-K^{*} v_{0}\right) \oplus\left(v+v_{0}\right)$. Since $\left(u-K^{*} v_{0}\right)+K^{*}\left(v+v_{0}\right)=u+K^{*} v=w$, this does not change $w$, but now

$$
\left(I+K K^{*}\right)\left(v+v_{0}\right)=K w+\left(I+K K^{*}\right) v_{0} \neq K w
$$

since $v_{0} \neq 0$ and $\left(I+K K^{*}\right)$ is injective. Thus also in this case we can find $g \in F_{2}$ such that

$$
\left\langle\left(I+K K^{*}\right)\left(v+v_{0}\right)-K w, g\right\rangle=\left\langle K^{*}\left(v+v_{0}\right), h\right\rangle .
$$

With this $g$ we put $f=h-K^{*} g$ in either case and deduce

$$
\langle w, h\rangle+\left\langle C_{1}^{*} w, C_{1}^{*} h\right\rangle=0
$$

for all $w \in\left(I, K^{*}\right)\left(D_{0}\right)$. Hence $h=0$ for all $h \in \mathcal{D}\left(C_{1}^{*}\right)$ if and only if $\left(I, K^{*}\right)\left(D_{0}\right) \cap$ $\mathcal{D}\left(C_{1}^{*}\right)$ is a core for $C_{1}^{*}$. Returning to (5.4) it follows (now for our original $f$ and $g$ ) that $h=0$ implies

$$
\langle u, f\rangle+\langle v, g\rangle=0
$$

for all $u \oplus v \in D_{0}$. Since $D_{0}$ is dense in $F_{1} \oplus F_{2}, f=0$ and $g=0$ follow.

Corollary 5.3. Let $C_{1}, C_{2}$ be such that $\mathcal{D}\left(C_{1}\right)=\mathcal{D}\left(C_{2}\right)$ and assume that $C_{1}$ is injective with $C_{2} C_{1}^{-1}$ having an extension $K \in \mathfrak{B}\left(F_{1}, F_{2}\right)$. Then $\overline{C^{\times}}=C^{*}$. 
Proof. For every $v_{0} \in \mathcal{D}\left(C_{1}^{*} K^{*}\right)$ we have $-K^{*} v_{0} \in \mathcal{D}\left(C_{1}^{*}\right)$, so that $\left(-K^{*} v_{0}, v_{0}\right) \in$ $\mathcal{D}\left(C^{\times}\right)$holds for every (and thus some) $v_{0} \in F_{2} \backslash\{0\}$ since $\mathcal{D}\left(C^{\times}\right)=\mathcal{D}\left(C_{1}^{*}\right) \oplus$ $\mathcal{D}\left(C_{1}^{*} K^{*}\right)$ by Proposition 4.6. Obviously, $\left(I, K^{*}\right)\left(\mathcal{D}\left(C^{\times}\right)\right) \supset \mathcal{D}\left(C_{1}^{*}\right)$, and an application of Proposition 5.2 completes the proof.

Example 5.4. Suppose $C$ is a column operator with $C_{2}=T-C_{1}$, where both $C_{1}$ and $T$ are in $\mathfrak{L}\left(F, F_{0}\right)$, and where $F \stackrel{\text { def }}{=} F_{1}=F_{2}$. Suppose moreover

$$
\mathcal{D}\left(C_{1}\right) \subset \mathcal{D}(T) \text { and } \mathcal{D}\left(C_{1}^{*}\right) \varsubsetneqq \mathcal{D}\left(T^{*}\right) .
$$

Then

$$
\mathcal{D}\left(C^{\times}\right) \varsubsetneqq \mathcal{D}\left(C^{*}\right) .
$$

Indeed, for $h \in \mathcal{D}\left(C_{1}\right), f, g \in F$,

$$
\langle C h, f \oplus g\rangle=\left\langle C_{1} h \oplus\left(T-C_{1}\right) h, f \oplus g\right\rangle=\left\langle C_{1} h, f-g\right\rangle+\langle T h, g\rangle .
$$

This gives us immediately that $f \oplus f$ is in $\mathcal{D}\left(C^{*}\right)$ if and only if $f$ is in $\mathcal{D}\left(T^{*}\right)$; then

$$
C^{*}(f \oplus f)=T^{*} f, \quad f \in \mathcal{D}\left(T^{*}\right)
$$

and consequently

$$
\mathcal{D}\left(C^{\times}\right) \subset \mathcal{D}\left(C^{\times}\right)+\left\{f \oplus f: f \in \mathcal{D}\left(T^{*}\right)\right\} \subset \mathcal{D}\left(C^{*}\right) .
$$

Hence, if $f \in \mathcal{D}\left(T^{*}\right) \backslash \mathcal{D}\left(C_{1}^{*}\right)$, then $f \oplus f \in \mathcal{D}\left(C^{*}\right)$ but $f \oplus f \notin \mathcal{D}\left(C_{1}^{*}\right) \oplus \mathcal{D}\left(C_{2}^{*}\right)=$ $\mathcal{D}\left(C^{\times}\right)$.

\section{THE OPERATOR MATRIX $A$}

Let $A$ be a $2 \times 2$ matrix of the form

$$
\left(\begin{array}{ll}
A_{11} & A_{12} \\
A_{21} & A_{22}
\end{array}\right)
$$

with the denseness condition (2.2) being satisfied. Writing

$$
C_{A, i}=\left(A_{i 1}, A_{i 2}\right), i=1,2,
$$

we know from Proposition 1 that

$$
C_{A, i}^{\prime}=\left(\begin{array}{c}
A_{i 1}^{\prime} \\
A_{i 2}^{\prime}
\end{array}\right)
$$

and thus

$$
A=\left(\begin{array}{l}
C_{A, 1} \\
C_{A, 2}
\end{array}\right), \quad A^{\times}=\left(C_{A, 1}^{\prime}, C_{A, 2}^{\prime}\right) .
$$

Theorem 6.1. $1^{\circ} A^{\times} \subset A^{\prime} .2^{\circ} A^{\times}$is closable. $3^{\circ}$ If $A^{\times}$is densely defined, then $A$ is closable.

Proof. $1^{\circ}$ immediately follows from (6.2) and Proposition 4.1. Since $A$ is densely defined, $A^{\prime}$ is a closed operator, and hence by $1^{\circ}, A^{\times}$is closable, which yields $2^{\circ}$. If $A^{\times}$is densely defined, so is $A^{\prime}$ by $1^{\circ}$. Consequently, $A$ is closable and $3^{\circ}$ follows.

Proposition 6.2. If $\mathcal{D}\left(C_{A, 1}^{\prime}\right) \oplus \mathcal{D}\left(C_{A, 2}^{\prime}\right)$ is dense in $F^{\prime}$, then $A$ is closable.

Proof. If $\mathcal{D}\left(C_{A, 1}^{\prime}\right) \oplus \mathcal{D}\left(C_{A, 2}^{\prime}\right)$ is dense in $F^{\prime}$, then $A^{\prime}$ is densely defined by part $1^{\circ}$ of Theorem [6.1, which means that $A$ is closable.

More can be said if at most one of the operators $A_{i j}$ is not bounded. 
Proposition 6.3. Assume that $A_{i j} \in \mathfrak{B}\left(E_{j}, F_{i}\right)$ with the exception of at most one pair $(i, j)$ and that this exceptional $A_{i j}$ is closable. Then $A$ is closable and $A^{\prime}=A^{\times}$.

Proof. By Proposition 3.5. both $C_{A, 1}$ and $C_{A, 2}$ are closable, and hence $A$ is closable by Proposition $4.32^{\circ}$. At most one of the operators $C_{A, i}$ in (6.1) does not satisfy $C_{A, i} \in \mathfrak{B}\left(E, F_{i}\right)$. Then Proposition 4.5 and (6.2) lead to $A^{\prime}=A^{\times}$.

Theorem 6.1 and Proposition 6.3 immediately raise the question if the following cases can occur:

I. $A$ is not closable,

II. $A$ is closable and $A^{\times}$is not densely defined,

III. $A^{\times}$is densely defined but $A^{\prime} \neq \overline{A^{\times}}$,

IV. $A^{\prime}=\overline{A^{\times}}$but $A^{\prime} \neq A^{\times}$.

Below we will show indeed that all these cases can occur, even under the additional requirement that all $A_{i j}$ are closed operators in Hilbert spaces.

Example 6.4. Here we give an example for I. Let $E_{1}=E_{2}=F_{1}=F_{2}=\ell^{2}$ and, with $R_{1}, R_{2}$ from Example 3.3, put $A_{11}=R_{1}, A_{12}=R_{2}, A_{21}=A_{22}=0$. Then all $A_{i j}$ are closed, and since $\left(R_{1}, R_{2}\right)$ is not closable, also $A$ is not closable by Proposition $4.32^{\circ}$.

Example 6.5. Here we give an example for II. Let $E_{1}=E_{2}=F_{1}=F_{2}=\ell^{2}$ and, with $R_{1}, R_{2}$ from Example 3.3 put $A_{11}=R_{1}, A_{12}=R_{2}, A_{21}=0, A_{22}=R_{2}$. Then all $A_{i j}$ are closed, $A$ is closable, while $A^{\times}$is not densely defined.

Proof. To show that $A$ is closable, consider any sequences

$$
f_{n} \in \mathcal{D}\left(A_{11}\right) \cap \mathcal{D}\left(A_{21}\right), g_{n} \in \mathcal{D}\left(A_{12}\right) \cap \mathcal{D}\left(A_{22}\right)
$$

satisfying $f_{n} \rightarrow 0, g_{n} \rightarrow 0, A_{11} f_{n}+A_{12} g_{n} \rightarrow h_{1}$ and $A_{21} f_{n}+A_{22} g_{n} \rightarrow h_{2}$ for some $h_{1}, h_{2}$ in $\ell^{2}$. Then

$$
h_{2}=\lim _{n \rightarrow \infty}\left(A_{21} f_{n}+A_{22} g_{n}\right)=\lim _{n \rightarrow \infty} R_{2} g_{n},
$$

$g_{n} \rightarrow 0$, and the closedness of $R_{2}$ imply that $h_{2}=0$. Consequently,

$$
h_{1}=\lim _{n \rightarrow \infty}\left(A_{11} f_{n}+A_{12} g_{n}\right)=\lim _{n \rightarrow \infty}\left(R_{1} f_{n}+R_{2} g_{n}\right)=\lim _{n \rightarrow \infty} R_{1} f_{n},
$$

so that $f_{n} \rightarrow 0$ and the closedness of $R_{1}$ imply $h_{1}=0$. This completes the proof of the closability of $A$. By Example 3.3. $\left(A_{11}, A_{12}\right)$ is not closable, whence, in view of Proposition 1, $\left(\begin{array}{l}A_{11}^{\prime} \\ A_{12}^{\prime}\end{array}\right)$ is not densely defined. Hence also $A^{\times}$is not densely defined.

Example 6.6. Here we give an example for III. Let $E_{1}=E_{2}=F_{1}=F_{2}=L_{2}(0,1)$ and let the operators $A_{i j}, i, j=1,2$ be defined by

$$
\begin{aligned}
\mathcal{D}\left(A_{11}\right) & =\left\{f \in W_{2}^{1}(0,1): f(0)=0\right\}, A_{11} f=f^{\prime}, \\
\mathcal{D}\left(A_{12}\right) & =L_{2}(0,1), A_{12}=0, \\
\mathcal{D}\left(A_{21}\right) & =\left\{f \in W_{2}^{1}(0,1): f(1)=0\right\}, A_{21} f=f^{\prime}, \\
A_{22} & =-A_{21},
\end{aligned}
$$

where $W_{2}^{1}(0,1)$ denotes the usual Sobolev space of order 1 . Then $A$ is densely defined, all $A_{i j}, i, j=1,2$, are closed, $A^{\times}$is densely defined, and $\overline{A^{\times}} \neq A^{\prime}$. 
Proof. The denseness of the domain of $A$ as well as the closedness of the $A_{i j}$ is well known and obvious. Since $A_{11}^{*}=-A_{21}$, it is also clear that $A^{\times}$is densely defined. Putting $C_{1}=\left(A_{11}, A_{12}\right), C_{2}=\left(A_{21}, A_{22}\right)$, we have

$$
A=\left(\begin{array}{l}
C_{1} \\
C_{2}
\end{array}\right) .
$$

We also let $C_{12}=\left.C_{1}\right|_{\mathcal{D}\left(C_{1}\right) \cap \mathcal{D}\left(C_{2}\right)}$ and $C_{21}=\left.C_{2}\right|_{\mathcal{D}\left(C_{1}\right) \cap \mathcal{D}\left(C_{2}\right)}$. Clearly,

$$
A=\left(\begin{array}{l}
C_{12} \\
C_{21}
\end{array}\right) \subset\left(\begin{array}{l}
\overline{C_{12}} \\
\overline{C_{21}}
\end{array}\right) \subset\left(\begin{array}{l}
\overline{C_{1}} \\
\overline{C_{2}}
\end{array}\right),
$$

and in view of Proposition $4.31^{\circ}$ it follows that

$$
\bar{A} \subset\left(\frac{\overline{C_{12}}}{\overline{C_{21}}}\right) \subset\left(\frac{\overline{C_{1}}}{\overline{C_{2}}}\right) .
$$

Hence, by Proposition $4.33^{\circ}$, the proof will be complete if we show that the second inclusion in (6.3) is strict, i. e.,

$$
\mathcal{D}\left(\overline{C_{12}}\right) \cap \mathcal{D}\left(\overline{C_{21}}\right) \neq \mathcal{D}\left(\overline{C_{1}}\right) \cap \mathcal{D}\left(\overline{C_{2}}\right) .
$$

Since $C_{2}(f \oplus f)=0$ for $f \in \mathcal{D}\left(A_{21}\right)$ we have $\left\{f \oplus f: f \in L_{2}(0,1)\right\} \subset \mathcal{D}\left(\overline{C_{2}}\right)$, which immediately leads to

$$
\left\{f \oplus f: f \in \mathcal{D}\left(A_{11}\right)\right\} \subset \mathcal{D}\left(\overline{C_{1}}\right) \cap \mathcal{D}\left(\overline{C_{2}}\right) .
$$

Since $A_{11}$ and $\left.A_{11}\right|_{\mathcal{D}\left(A_{11}\right) \cap \mathcal{D}\left(A_{21}\right)}$ are closed operators, so are $C_{1}$ and $C_{12}$. Hence $f \oplus g \in \mathcal{D}\left(\overline{C_{12}}\right) \cap \mathcal{D}\left(\overline{C_{21}}\right)$ implies $f \in \mathcal{D}\left(A_{11}\right) \cap \mathcal{D}\left(A_{21}\right)$, and thus (6.4) is proved in view of (6.5) and $\mathcal{D}\left(A_{11}\right) \not \subset \mathcal{D}\left(A_{21}\right)$.

Example 6.7. Here we give an example for IV. Let $E_{1}=E_{2}=F_{1}=F_{2}$ be an infinite-dimensional Hilbert space and let $A_{11}$ be a closed densely defined unbounded operator in this Hilbert space. Put $A_{12}=A_{22}=0$ and $A_{21}=-A_{11}$. Then $A$ is a densely defined closed operator, $\overline{A^{\times}}=A^{*}$, and $A^{\times} \neq A^{*}$.

Indeed, the operator $A$ is clearly densely defined and closed, and

$$
A^{\times}=\left(\begin{array}{cc}
A_{11}^{*} & -A_{11}^{*} \\
0 & 0
\end{array}\right) .
$$

Considering $A^{\times}$as a column operator of row operators (one may look also at Theorem 10 in [15) and applying Propositions 4.5 and 1 we come to

$$
A^{\times *}=\left(\begin{array}{cc}
A_{11}^{* *} & 0 \\
-A_{11}^{* *} & 0
\end{array}\right)=A
$$

since $A_{11}$ is closed. Taking adjoints gives $\overline{A^{\times}}=A^{*}$. $A^{*}$.

Using Example 5.4 with $T=0$ we get $\mathcal{D}\left(A^{\times}\right) \neq \mathcal{D}\left(A^{*}\right)$, which establishes $A^{\times} \neq$

\section{REFERENCES}

[1] F. V. Atkinson, H. Langer, R. Mennicken, A. A. Shkalikov, The essential spectrum of some matrix operators, Math. Nachr. 167 (1994), 5-22. MR1285306 (95f:47007)

[2] J. A. Burns, T. L. Herdman, Adjoint semigroup theory for a class of functional differential equations, SIAM J. Math. Anal. 7 (1976), 729-745. MR0417528 (54:5578)

[3] W. Desch, R. Grimmer, W. Schappacher, Wellposedness and wave propagation for a class of integrodifferential equations in Banach space, J. Diff. Equ. 74 (1988), 391-411. MR952904 (89e:45012) 
[4] K.-L. Engel, Matrix representation of linear operators on product spaces, Rend. Circ. Mat. Palermo (2) Suppl. 56 (1998), 219-224. MR1710840(2000f:47004)

[5] J. P. Friedberg, Ideal Magnetohydrodynamics, Plenum Press, New York, 1987.

[6] V. Hardt, A. Konstantinov, R. Mennicken, S. Naboko, On the spectrum of the product of closed operators, Math. Nachr 215 (2000), 91-102. MR1768195(2001b:47008)

[7] V. Hardt, R. Mennicken, A. K. Motovilov, A factorization theorem for the transfer function associated with a $2 \times 2$ operator matrix having unbounded couplings, J. Operator Theory 48 (2002), no. 1, 187-226. MR.1926050 (2003g:47026)

[8] K. Hain, R. Lüst, Zur Stabilität zylindersymmetrischer Plasmakonfigurationen mit Volumenströmen, Z. Naturforsch. A 13 (1958), 936-940. MR0106671(21:5402)

[9] S. Hassi, A. Sandovici, H. de Snoo and H. Winkler, Extremal extensions for the sum of nonnegative selfadjoint relations, Proc. Amer. Math. Soc., 135 (2007), 3193-3204. MR 2322750

[10] A. Konstantinov, R. Mennicken, On the Friedrichs extension of some block operator matrices, Integral Equations Operator Theory 42 (2002), 472-481. MR.1885445 (2003m:47039)

[11] P. Kurasov, S. Naboko, On the essential spectrum of a class of singular matrix differential operators. I: Quasiregularity conditions and essential self-adjointness, Mathematical Physics, Analysis and Geometry 5 (2002), 243-286. MR1940117 (2003m:47084)

[12] A. Lifschitz, Magnetohydrodynamics and Spectral Theory, Kluwer, Dordrecht, 1989. MR.990647 (90k:76097)

[13] R. Nagel, Towards a "matrix theory" for unbounded operator matrices, Math. Z. 201 (1989), 57-68. MR990188 (90c:47004)

[14] S. Ôta, K. Schmüdgen, Some selfadjoint $2 \times 2$ operator matrices associated with closed operators, Integr. Equ. Oper. Theory 45 (2003), 475-484. MR.1971749 (2004b:47041)

[15] F.H. Szafraniec, On normal extensions of unbounded operators: IV. A matrix construction, in: Operator Theory: Adv. Appl. 163 (2005), 337-350. MR2215869 (2007b:47052)

The John Knopfmacher Centre for Applicable Analysis and Number Theory, School of Mathematics, University of the Witwatersrand, WitS, 2050, South Africa

E-mail address: manfred@maths.wits.ac.za

Instytut Mathematyki, Uniwersytet Jagielloński, ul. Reymonta 4, Pl-30059 Kraków, POLAND

E-mail address: fhszafra@im.uj.edu.pl 